\title{
BMPR2 is required for postimplantation uterine function and pregnancy maintenance
}

\author{
Takashi Nagashima, ${ }^{1}$ Qinglei Li,2 Caterina Clementi, ${ }^{1,3}$ John P. Lydon,, ${ }^{4,5}$ \\ Francesco J. DeMayo, ${ }^{3,4,5,6}$ and Martin M. Matzuk',3,4,5,6,7,8
}

\begin{abstract}
1Department of Pathology and Immunology, Baylor College of Medicine, Houston, Texas, USA. 2Department of Veterinary Integrative Biosciences, College of Veterinary Medicine and Biomedical Sciences, Texas A\&M University, College Station, Texas, USA. ${ }^{3}$ Program in Developmental Biology, ${ }^{4}$ Department of Molecular and Cellular Biology, ${ }^{5}$ Center for Reproductive Medicine, ${ }^{6}$ Center for Drug Discovery, ${ }^{7}$ Department of Molecular and Human Genetics, and ${ }^{8}$ Department of Pharmacology, Baylor College of Medicine, Houston, Texas, USA.
\end{abstract}

\begin{abstract}
Abnormalities in cell-cell communication and growth factor signaling pathways can lead to defects in maternal-fetal interactions during pregnancy, including immunologic rejection of the fetal/placental unit. In this study, we discovered that bone morphogenetic protein receptor type 2 (BMPR2) is essential for postimplantation physiology and fertility. Despite normal implantation and early placental/fetal development, deletion of Bmpr2 in the uterine deciduae of mice triggered midgestation abnormalities in decidualization that resulted in abnormal vascular development, trophoblast defects, and a deficiency of uterine natural killer cells. Absence of BMPR2 signaling in the uterine decidua consequently suppressed IL-15, VEGF, angiopoietin, and corin signaling. Disruption of these pathways collectively lead to placental abruption, fetal demise, and female sterility, thereby placing BMPR2 at a central point in the regulation of several physiologic signaling pathways and events at the maternal-fetal interface. Since trophoblast invasion and uterine vascular modification are implicated in normal placentation and fetal growth in humans, our findings suggest that abnormalities in uterine BMPR2mediated signaling pathways can have catastrophic consequences in women for the maintenance of pregnancy.
\end{abstract}

\section{Introduction}

In the first trimester of mammalian pregnancy, embryo implantation, decidualization, and vascular modification, including spiral artery remodeling, are key events for the establishment of a successful pregnancy. Implantation is the physiological and immunological acceptance between the conceptus and the maternal uterine endometrium. During implantation, the endometrium undergoes morphological and physiological changes to accommodate the conceptus $(1,2)$, and the endometrial extracellular matrix undergoes degradation to permit embryo invasion (3). Decidualization is the process of uterine stromal cell proliferation and differentiation in response to the implanting extraembryonic/embryonic unit and is essential for supporting embryonic growth before establishment of the placenta (4). Uterine spiral artery remodeling is a maternal adaptation process and is critical for the establishment of a fully functional placental unit. During spiral artery remodeling, fetal trophoblast cells invade the decidua and its blood vessels, and uterine spiral arteries transform from narrow to large canals to increase maternal blood flow toward the implantation sites and to facilitate the increasing demands of the fetus for nutrition and oxygen (5, 6). Failures in this process (e.g., shallow trophoblast invasion and incomplete remodeling) cause reduced blood flow to the fetoplacental unit and are associated with common pregnancy complications, such as recurrent miscarriages, fetal growth retardation, and preeclampsia $(7,8)$. However, the mechanisms underlying decidual vascular formation and remodeling are yet to be elucidated.

Bone morphogenetic proteins (BMPs), the largest group of the TGF- $\beta$ superfamily, are pleiotropic growth factors that were originally isolated from the bone as proteins that induce bone and cartilage formation $(9,10)$. BMPs regulate a wide variety of cellu-

Conflict of interest: The authors have declared that no conflict of interest exists. Citation for this article: J Clin Invest. 2013;123(6):2539-2550. doi:10.1172/JCI65710. lar functions and play critical roles in mammalian development and reproductive function (11-13). BMP ligands signal through a heteromeric complex of type 1 and type 2 serine/threonine kinase receptors (11). There are $3 \mathrm{BMP}$ type 2 receptors (BMP receptor type 2 [BMPR2]), activin receptor type $2 \mathrm{~A}$ (ACVR2A), and activin receptor type $2 \mathrm{~B}$ (ACVR2B) and 3 type 1 receptors (activin receptor-like kinase 2, 3, and 6). Ligand binding to the BMP type 2 and type 1 receptors leads to phosphorylation of SMAD1, SMAD5, and SMAD8, which then complex with SMAD4 to regulate target gene transcription in the nucleus (11).

The potential importance of BMPR2 in BMP signaling during vasculogenesis is highlighted by the findings that mutations in the BMPR2 gene in humans lead to haploinsufficiency and cause primary pulmonary hypertension (14). In mice, Bmpr2 homozygous null mutants die during gastrulation without forming mesoderm (15), while mice homozygous for a Bmpr2 hypomorphic allele, which lacks the ligand-binding domain, die at midgestation and exhibit skeletal and cardiovascular abnormalities (16). The early embryonic lethality associated with loss of BMPR2 function precludes functional analysis of its roles in the female reproductive system. To overcome this difficulty and define the in vivo roles of BMPR2 in the female reproductive system, we generated Bmpr2 conditional knockout (cKO) mice using mice with a knockin of the cre recombinase gene into the progesterone receptor $(P g r)$ locus and examined critical events during the course of pregnancy. Whereas mice with a conditional deletion of BMP2, one of the ligands for BMPR2, demonstrate a defect in uterine decidualization at the beginning of pregnancy (17), Bmpr2 cKO female mice initially demonstrate normal implantation and placental and fetal growth. However, as the pregnant $B m p r 2 \mathrm{cKO}$ female mice approach midgestation, there is severe hemorrhage at the implantation sites and fetal growth retardation, leading to placental abruption, fetal demise, 
A

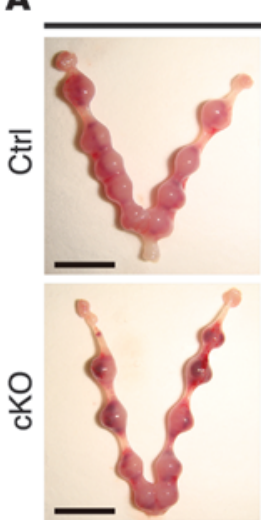

E9
Implantation sites

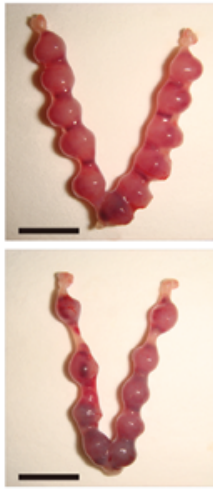

E10

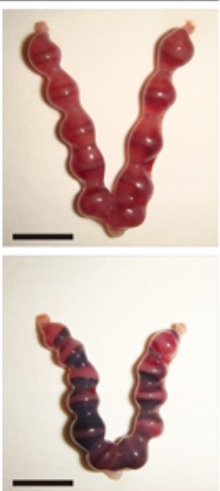

E11
B

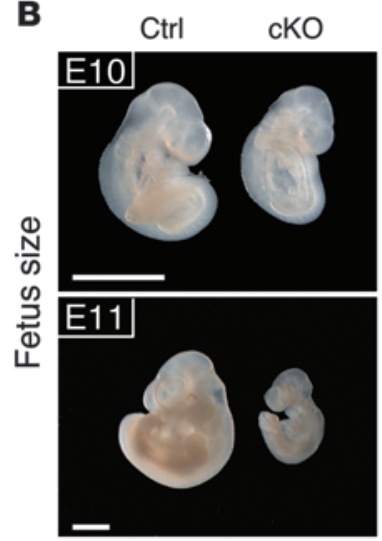

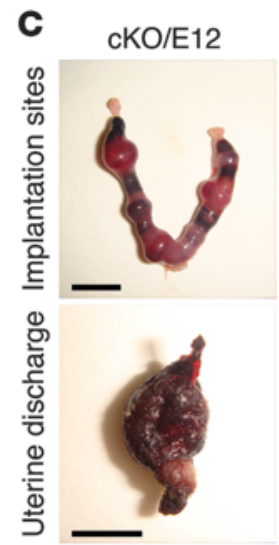

D

H\&E/E9
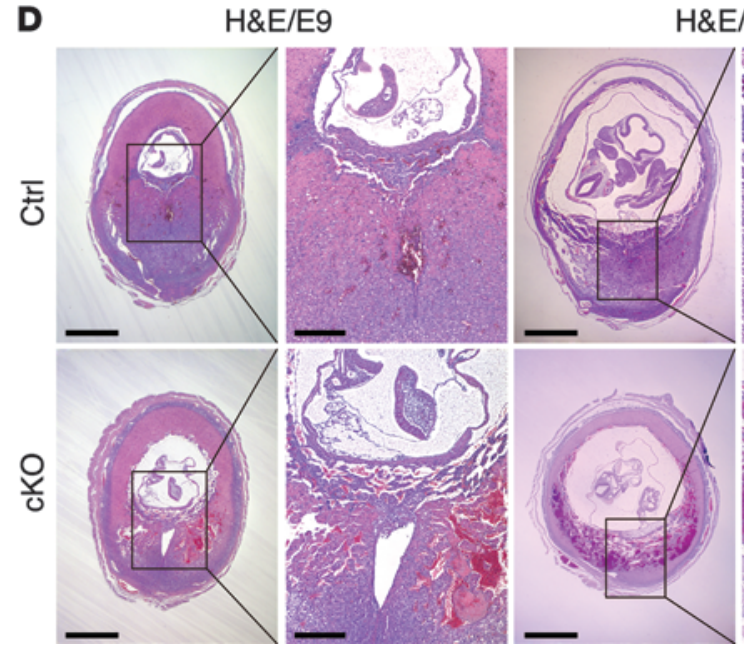

$\mathrm{H} \& \mathrm{E} / \mathrm{E} 10$
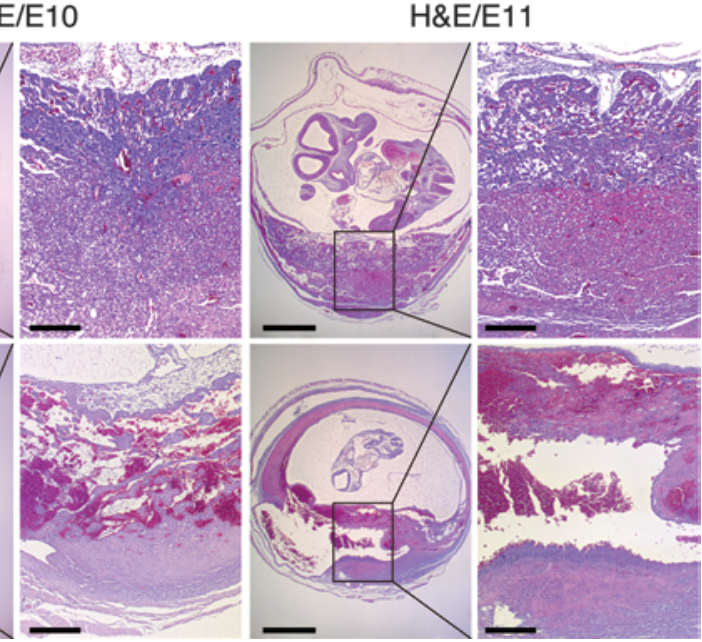

Figure 1

Decidual growth restriction, fetal growth retardation, and hemorrhagic implantation sites in pregnant $B m p r 2 \mathrm{cKO}$ female mice. (A) Hemorrhagic implantation sites in pregnant Bmpr2 cKO female mice. Scale bar: $1 \mathrm{~cm}$. (B) Fetal growth retardation in Bmpr2 cKO female mice is grossly obvious at E10 and E11. Scale bar: $1 \mathrm{~mm}$. (C) Uterine hemorrhage and discharge of fetus and placenta in Bmpr2 cKO female mice at E12. Scale bar: $1 \mathrm{~cm}$ (top); $5 \mathrm{~mm}$ (bottom). (D) Hemorrhagic implantation sites and fetal growth retardation were detected in Bmpr2 cKO female mice beginning at E9. All sections were stained with H\&E. Higher-magnification images of the boxed regions are shown at right. Scale bar: $1 \mathrm{~mm}$ (lower magnification); $300 \mu \mathrm{m}$ (higher magnification).

and sterility. These findings implicate BMPR2-mediated signaling as a major pathway that regulates the maternal-fetal interface during pregnancy in women.

\section{Results}

Generation of Bmpr2 $c K O$ mice and localization of BMPR2 to uterine decidua. Because of embryonic lethality in Bmpr2-null mice $(15,16)$, we generated a Bmpr2 cKO mouse model using Pgr-cre and a Bmpr2 floxed allele (Supplemental Figure 1A; supplemental material available online with this article; doi:10.1172/ JCI65710DS1) to define the physiological roles of BMPR2 in female reproduction. Pgr-cre is expressed postnatally at high levels in the anterior lobe of the pituitary and epithelial and stromal compartments of the uterus and granulosa cells of the preovulatory follicles in the ovary $(18,19)$. To achieve maximal deficiency of Bmpr2, the Bmpr2-null allele was also used in the breeding scheme (ref. 15 and Supplemental Figure 1B). Twomonth-old control (Bmpr2fl--) and Bmpr2 cKO (Bmpr $\left.2^{f l-P g r c r e /+}\right)$ female mice were confirmed to have efficient deletion of uterine Bmpr2 (Supplemental Figure 1, C and D), and the Bmpr2 mRNA and protein levels were statistically lower in $B m p r 2 \mathrm{cKO}$ uteri (Supplemental Figure 1, E and F). Immunofluorescence analysis of E8 implantation sites localized BMPR2 expression to the cell membranes of mesometrial decidual cells and uterine smooth muscle cells in control female mice (Supplemental Figure 1G). Consistent with sites of high cre expression in the Pgr-cre-positive female mice, the Bmpr2 cKO mice demonstrated loss of BMPR2 in the deciduae but not uterine smooth muscle (Supplemental Figure 1G). Significant BMPR2 expression was not observed in developing vasculature (data not shown).

Bmpr2 cKO female mice are sterile but display normal ovarian functions, embryo development, and implantation. To evaluate the fertility of Bmpr2 cKO female mice, we conducted a breeding study in which sexually mature control and Bmpr2 cKO female mice ( $n=10$ for each genotype) at 6 weeks were mated with known fertile WT male mice for 6 months. Whereas control female mice 
A



D

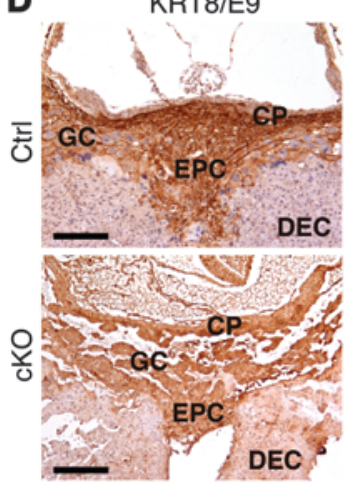

B

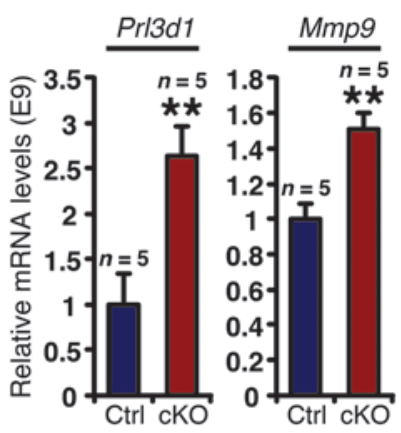

C

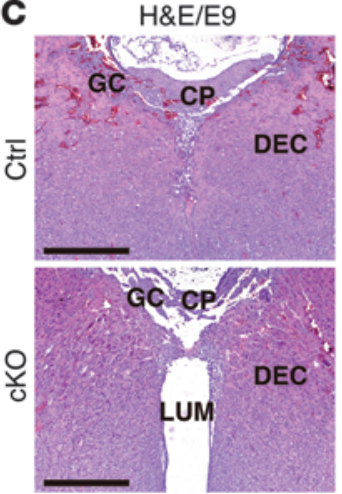

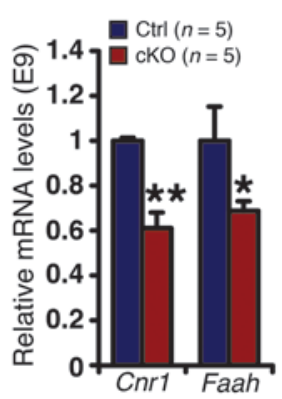

F
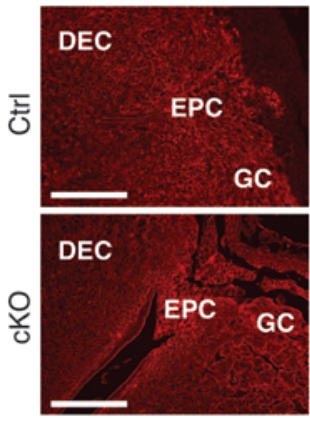

TUNEL/E9

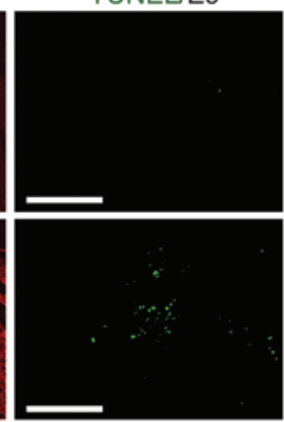

DAPI/Merge/E9

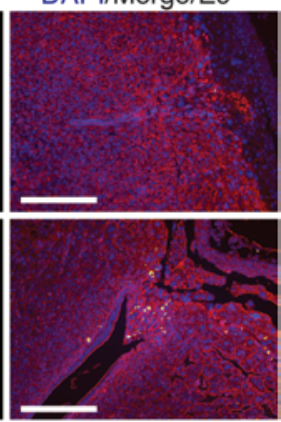

G

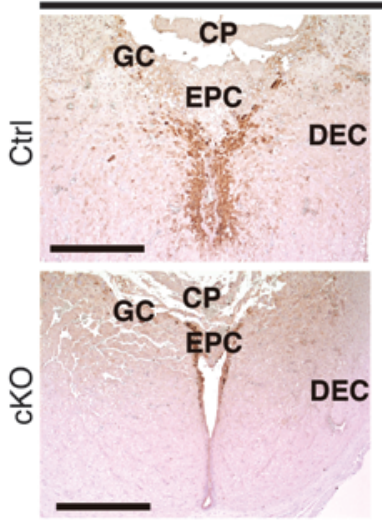

PTGS2/E9

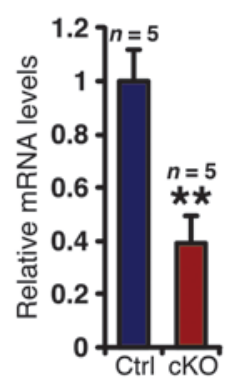

H

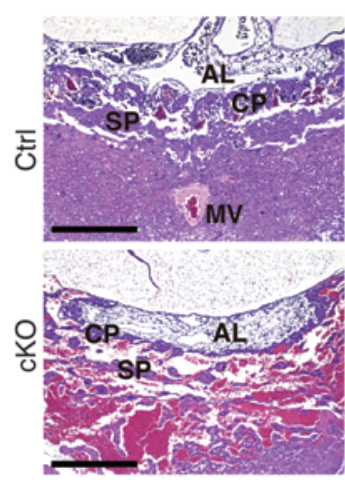

laminin/E10



Figure 2

Increased trophoblast giant cells, regressed formation of ectoplacental cone, and failure of fetal blood vessel formation in implantation sites of Bmpr2 cKO female mice. (A) Deletion of uterine Bmpr2 induces an increase of PRL3D1-positive trophoblast giant cells and disruption of the normal structure of the layers. CP, chorionic plate; EPC, ectoplacental cone; GC, trophoblast giant cells; DEC, decidua. Scale bar: $500 \mu \mathrm{m}$ (left); $200 \mu \mathrm{m}$ (right). (B) Deletion of uterine Bmpr2 increases Prl3d1 and Mmp9 mRNA levels in E9 trophoblast cells ( ${ }^{\star \star} P<0.01$ ). Data are mean \pm SEM. (C and $\mathbf{D})$ Regression of the ectoplacental cone and failure to compress the uterine lumen (LUM) in E9 implantation sites of Bmpr2 cKO uteri. KRT8, cytokeratin 8. Scale bar: $500 \mu \mathrm{m}$ (C); $200 \mu \mathrm{m}$ (D). (E) Deletion of uterine Bmpr2 decreases Cnr1 and Faah mRNA levels in E9 trophoblast cells $\left({ }^{*} P<0.05 ;{ }^{* *} P<0.01\right)$. Data are mean \pm SEM. (F) Increased apoptosis was detected in E9 invasive trophoblast cells from Bmpr2 cKO uteri by TUNEL assay. Scale bar: 500 um. (G) Impaired trophoblast invasion was detected in E9 implantation sites of Bmpr2 cKO uteri by immunostaining for PTGS2 and real-time quantitative PCR for Ptgs2 mRNA (** $P<0.01$ ). Scale bar: $500 \mu \mathrm{m}$. Data are mean \pm SEM. (H) Impaired formation of fetal blood vessels was detected in E10 implantation sites of Bmpr2 cKO uteri. Arrowheads indicate fetal blood vessels. AL, allantois; SP, spongiotrophoblast cells; FV, fetal blood vessel; MV, maternal blood vessel. Scale bar: $500 \mu \mathrm{m}$ (left and middle); $200 \mu \mathrm{m}$ (right). (A and $\mathbf{H}$ ) Higher-magnification images of the boxed regions are shown at right. 
A



C

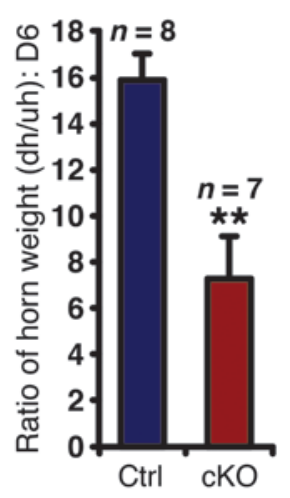

Decidualization protocol

D

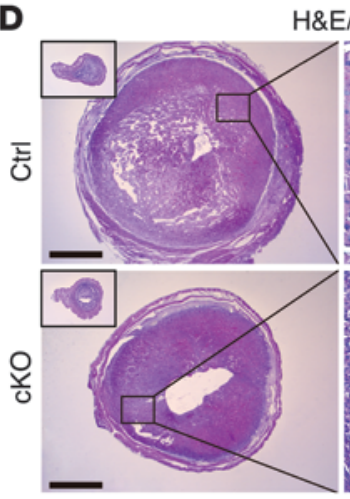

B

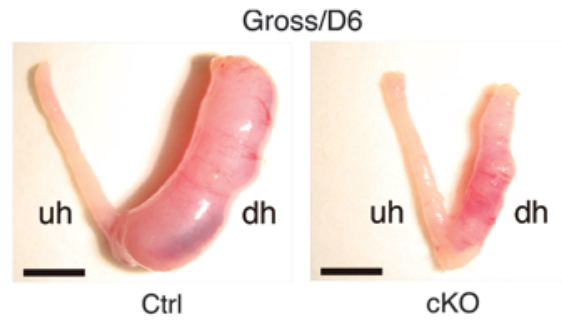

E

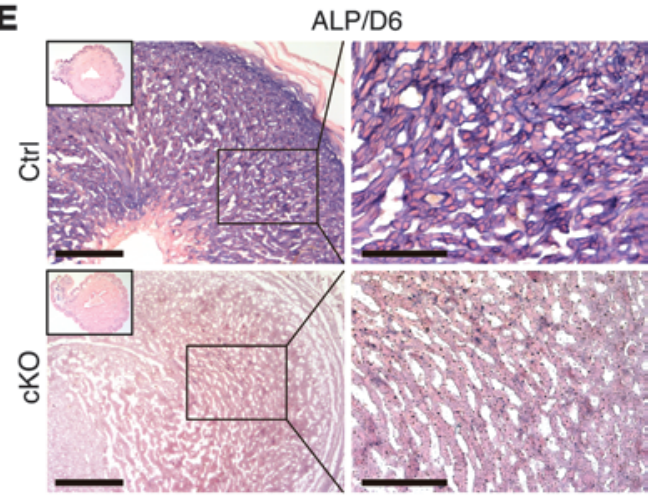

$\mathbf{F}$

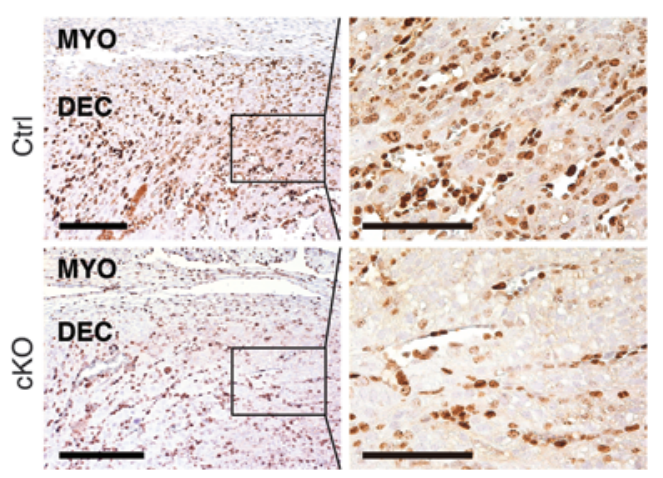

G

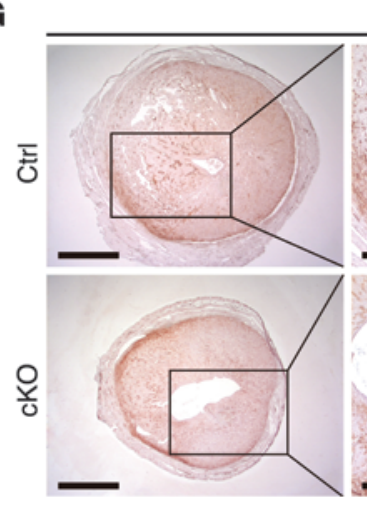

PECAM1/D6

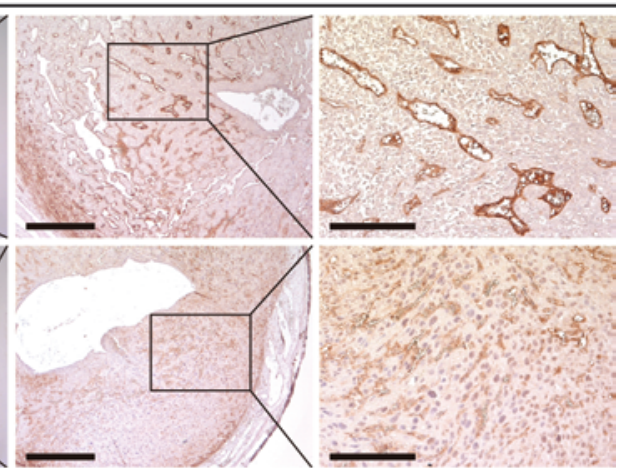

\section{Figure 3}

Differentiation, proliferation, and vascularization defects in artificially decidualized Bmpr2 cKO female mice. (A) Representation of the artificial decidualization procedure. (B and C) Gross morphology of uterine horns with or without decidual stimulation. Decidual responses were reduced in stimulated Bmpr2 cKO uterine horns, including decreased weights ( $\left.{ }^{* *} P<0.01\right)$. uh, unstimulated horn; dh, decidualized horn; D6, day 6 after artificial decidual stimulation. Scale bar: $5 \mathrm{~mm}$. Data are mean \pm SEM. (D) Stimulated Bmpr2 cKO uterine horns showed suppressed transformation from fibroblasts to epithelioid cells. The insets show unstimulated uterine horn morphology. Scale bar: $1 \mathrm{~mm}$ (left); $100 \mu \mathrm{m}$ (right). (E) Alkaline phosphatase (ALP) activity, a marker for stromal cell differentiation in response to decidualization, was reduced in stimulated Bmpr2 cKO uterine horns. The dark blue deposits indicate ALP-positive activity, and the nuclei were counterstained with nuclear fast red. The insets show lower ALP activity in unstimulated uterine horns. Scale bar: $200 \mu \mathrm{m}$. (F) Decreased decidual cell proliferation was detected in stimulated Bmpr2 cKO uterine horns by immunostaining for MKI67. MYO, myometrium. Scale bar: $400 \mu \mathrm{m}$ (left); $100 \mu \mathrm{m}$ (right). (G) Lack of decidual blood vessels in stimulated Bmpr2 cKO uterine horns was revealed by immunostaining against PECAM1 for detection of vascular endothelial cells. Scale bar: $1 \mathrm{~mm}$ (left); $500 \mu \mathrm{m}$ (middle); $200 \mu \mathrm{m}$ (right). (D-G) Higher-magnification images of the boxed regions are shown at right.

demonstrated normal fertility and fecundity $(8.8 \pm 0.4$ pups per litter and $1.1 \pm 0.0$ litter per month) compared with that of WT mice $(8.1 \pm 0.3$ pups per litter and $1.2 \pm 0.1$ litter per month) and $\mathrm{Pg}_{\mathrm{g}} \mathrm{rre}^{+}$female mice $(7.8 \pm 0.1$ pups per litter and $1.1 \pm 0.4$ litter per month), Bmpr2 cKO female mice never delivered any live pups, revealing that BMPR2 is essential for female fertility. Vaginal plugs were found in $\mathrm{Bmpr} 2 \mathrm{cKO}$ female mice, indicating that the infertility was not due to disrupted mating behavior. Bmpr2 cKO female mice also demonstrated normal hormone levels
(Supplemental Table 1), ovarian and uterine histology, follicle growth, ovulation, fertilization, and preimplantation embryo development (Supplemental Figure 2). Our findings indicate that the infertility phenotype in $B m p r 2 \mathrm{cKO}$ female mice is not caused by defects in ovarian functions and embryo development but is likely caused by uterine defects.

We next investigated the effects of uterine Bmpr2 deletion on the implantation process. Spatiotemporal and reciprocal interactions between the blastocyst and the receptive endometrium 
A

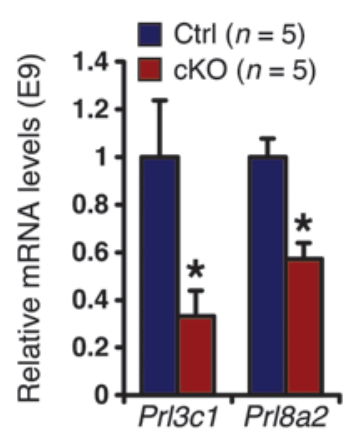

C

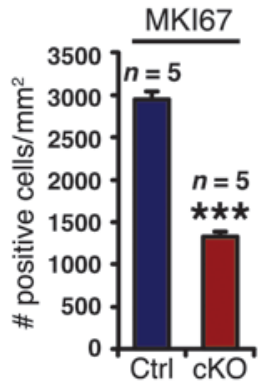

B

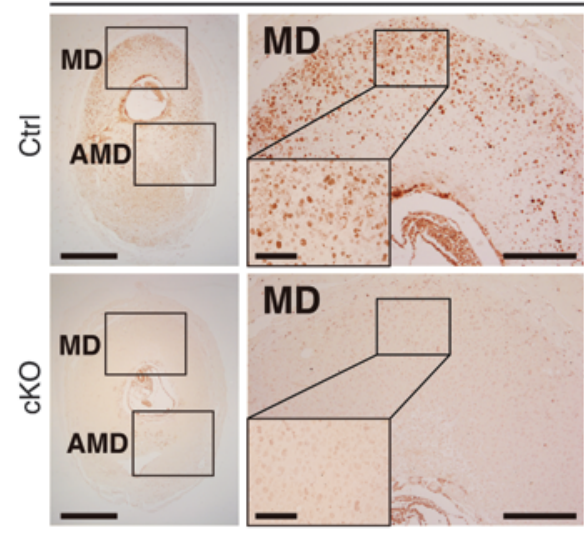

E9

D
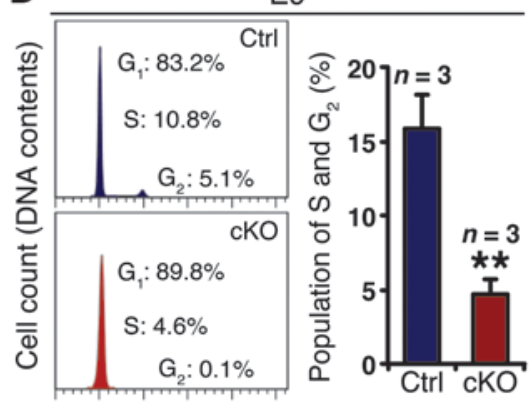
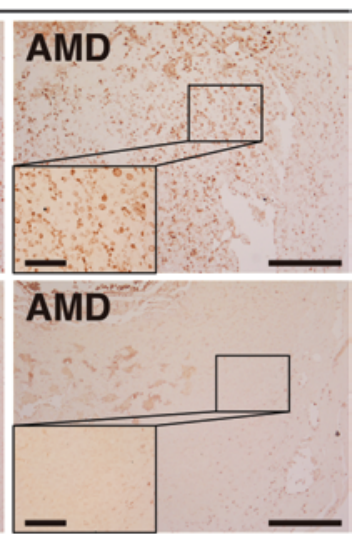

E

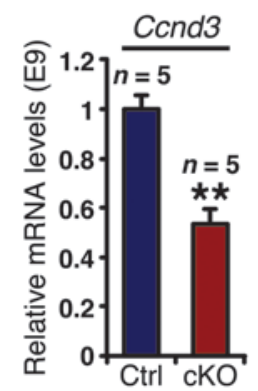

F

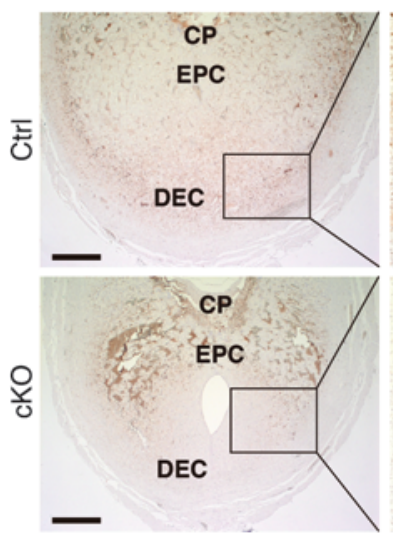

CCND3/E9

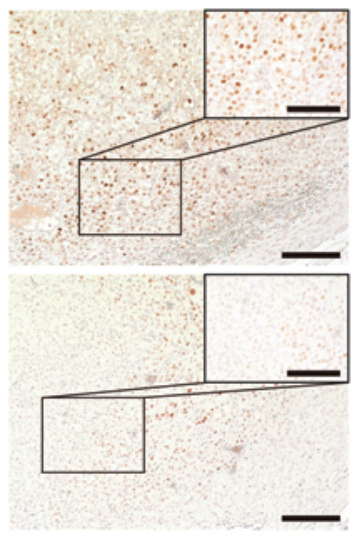

G

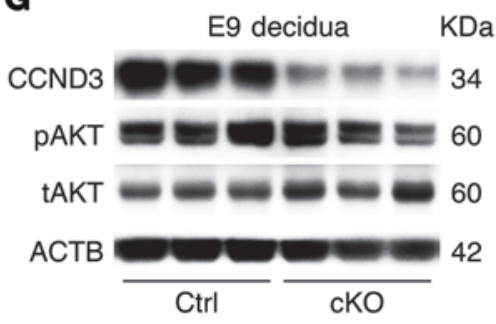

Figure 4

Decidual cell proliferation defects in uteri of pregnant Bmpr2 cKO mice. (A) Deletion of uterine Bmpr2 decreases Prl3c1 and Prl8a2 mRNA levels in E9 decidual cells ( $\left.{ }^{*} P<0.05\right)$. Data are mean \pm SEM. (B and $\mathbf{C}$ ) Restricted decidual cell proliferation was detected in both MD and AMD compartments within E9 implantation sites of Bmpr2 cKO uteri by counting MKI67-positive cells without counterstaining (*** $P<0.001)$. Scale bar: $1 \mathrm{~mm}$ (left); $300 \mu \mathrm{m}$ (middle and right); $100 \mu \mathrm{m}$ (insets). Data are mean \pm SEM. (D) Representative histograms of flow cytometric analyses and calculated populations of $S / G_{2}$ phase cells in isolated decidual cells from $E 9$ implantation sites. Increased $G_{1}$ phase and decreased $S / G_{2}$ phase population in E9 implantation sites of Bmpr2 cKO uteri ( $\left.{ }^{\star \star} P<0.01\right)$. Data are mean \pm SEM. (E) Deletion of uterine $B m p r 2$ decreases Ccnd3 mRNA levels in E9 trophoblast cells $\left({ }^{\star *} P<0.01\right)$. Data are mean \pm SEM. (F) Reduced CCND3-positive cells were detected in E9 implantation sites of Bmpr2 cKO uteri without counterstaining. Scale bar: $500 \mu \mathrm{m}$ (left); $300 \mu \mathrm{m}$ (right); $100 \mu \mathrm{m}$ (inset). (G) Western blot analysis of isolated E9 decidual cells from Bmpr2 cKO uteri shows decreased expression of CCND3 but not PAKT and tAKT. ACTB expression was used as an internal control. ( $\mathbf{B}$ and $\mathbf{F})$ Higher-magnification images of the boxed regions are shown at right, and within these images, higher-magnification images of the boxed regions are shown as insets. 
A
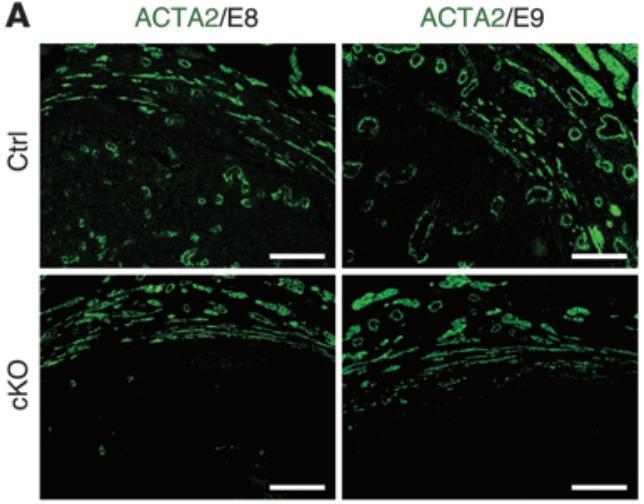

B

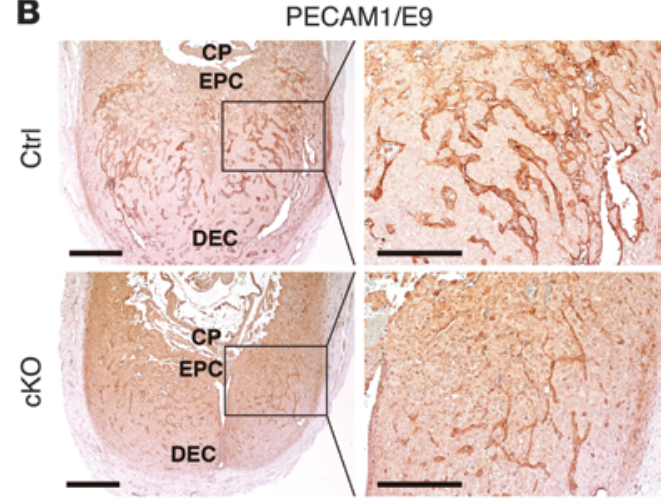

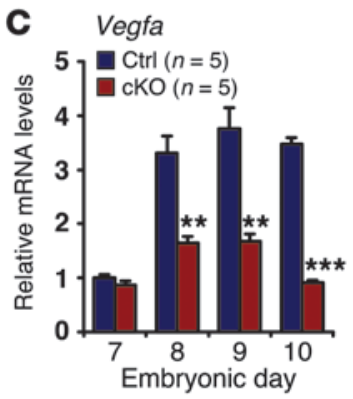
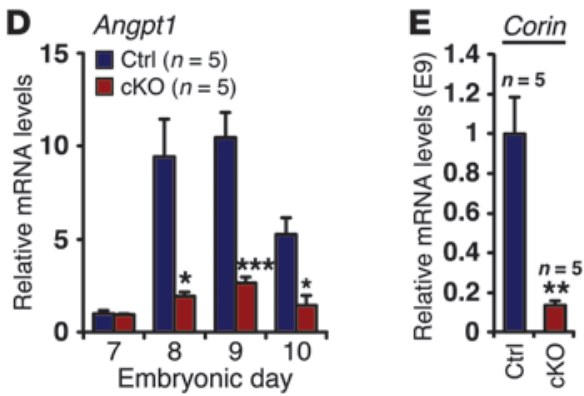

\section{Figure 5}

Impaired uterine decidual vascularization and decreased VEGF, ANGPT, and CORIN expression in the deciduae of pregnant cKO female mice. (A) Decreased vessels in the deciduae of pregnant Bmpr2 cKO mice at E8 and E9, as revealed by immunofluorescence with an antibody against ACTA2. Scale bar: $200 \mu \mathrm{m}$. (B) The narrow lumen of decidual blood vessels in E9 implantation sites of $B m p r 2 \mathrm{cKO}$ uteri was revealed by immunostaining against PECAM1 for detection of vascular endothelial cells. Higher-magnification images of the boxed regions are shown at right. Scale bar: $500 \mu \mathrm{m}$. (C-E) Deletion of uterine Bmpr2 decreases (C) Vegfa, (D) Angpt1, and (E) Corin mRNA levels ( ${ }^{*} P<0.05$; $\left.{ }^{\star \star} P<0.01 ;{ }^{* \star} P<0.001\right)$. Data are mean \pm SEM.

are required for normal implantation (20). The attachment reaction coincides with increased stromal vascular permeability at the site of the blastocyst and can be analyzed with Chicago blue injection (21). Using this method, the number, weight, and histology of E5 implantation sites and molecular analyses of key uterine implantation genes were found to be comparable between control and Bmpr2 cKO female mice (Supplemental Figure 3). Thus, in contrast to that in $B m p 2 \mathrm{cKO}$ mice (17), uterine BMPR2 is not essential for implantation.

Decidual growth restriction, fetal growth retardation, and hemorrbagic implantation sites in pregnant Bmpr2 $c K O$ female mice. The decidual structure is essential for provision of nutrition to the developing embryo and acts as a barrier against uncontrolled trophoblast proliferation, allowing normal placentation by controlling hypoxia signaling pathways $(22,23)$. Until E8, uterine morphology, embryo development, and extraembryonic structures appeared grossly and histologically comparable between control and $B m p r 2$ cKO female mice, and mRNA and protein levels of PRL3D1 and MMP9, markers for trophoblast giant cells, were normal (Supplemental Figure 4). However, beginning at $\mathrm{E} 9$, we found hemorrhage in the implantation sites of Bmpr2 cKO uteri (Figure 1, A and D). Although there were similar numbers of implantation sites (control, $8.1 \pm 1.3$ per uterus [ $n=7] ; \mathrm{Bmpr} 2 \mathrm{cKO}, 7.4 \pm 1.0$ per uterus $[n=7]$ ), the weights of the implantation sites trended lower at E6 to E8 and were significantly lower in Bmpr2 cKO female mice at E9 to E11 (Supple- mental Figure $5 \mathrm{~A}$ ), suggestive of alterations in decidual cell growth and/or fetal growth retardation. Uterine Bmpr2 mRNA levels also trended higher between E5 and E8 and were statistically elevated at E9 to E11 (Supplemental Figure 5B). Paralleling these findings, E10 and E11 fetuses from Bmpr2 cKO female mice have obvious growth retardation (Figure 1, B and D), and some pregnant Bmpr2 cKO female mice discharged the hemorrhagic implantation tissue at E12 (Figure 1C). The hemorrhage was limited to part of the trophoblast and decidual tissues at E9 but then became more extensive and severe at E10 and E11, concomitant with disappearance of the normal structure of the trophoblast and decidual cell layers (Figure 1D). These data demonstrate that signaling through BMPR2 is required at the maternal-fetal interface.

Normal steroid hormone levels during pregnancy. Because ovarian hormones control pregnancy progression, including decidual growth, parturition, and pregnancy outcome, we measured serum $17 \beta$-estradiol $\left(\mathrm{E}_{2}\right)$ and progesterone $\left(\mathrm{P}_{4}\right)$ levels in pregnant control and Bmpr2 cKO female mice as well as their receptor mRNAs in decidual tissue. Serum hormone levels were comparable between pregnant control and Bmpr2 cKO female mice (Supplemental Figure 6, A and B), and mRNAs of Esr1 and Pgr (receptors for $\mathrm{E}_{2}$ and $\mathrm{P}_{4}$ ) were similar in pregnant control and Bmpr2 cKO uteri (Supplemental Figure 6, C and D). Protein levels of $3 \beta$-HSD, which catalyzes the synthesis of $\mathrm{P}_{4}$ from pregnenolone, were also similar in control and $B m p r 2 \mathrm{cKO}$ ovaries at 
A
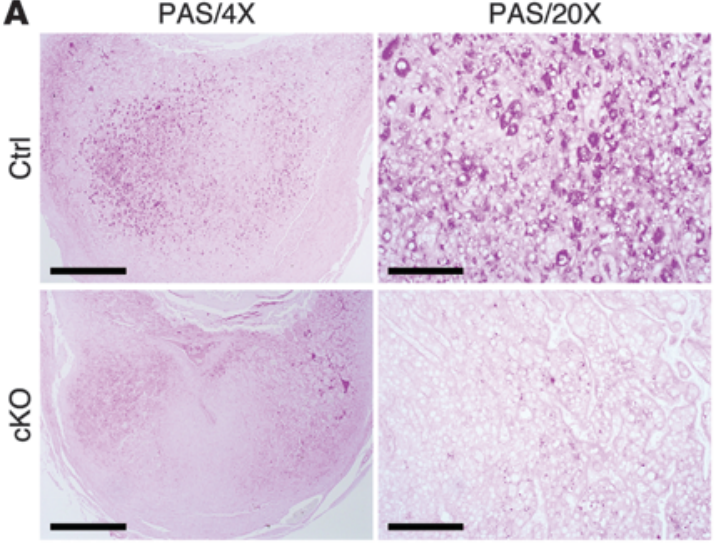

B
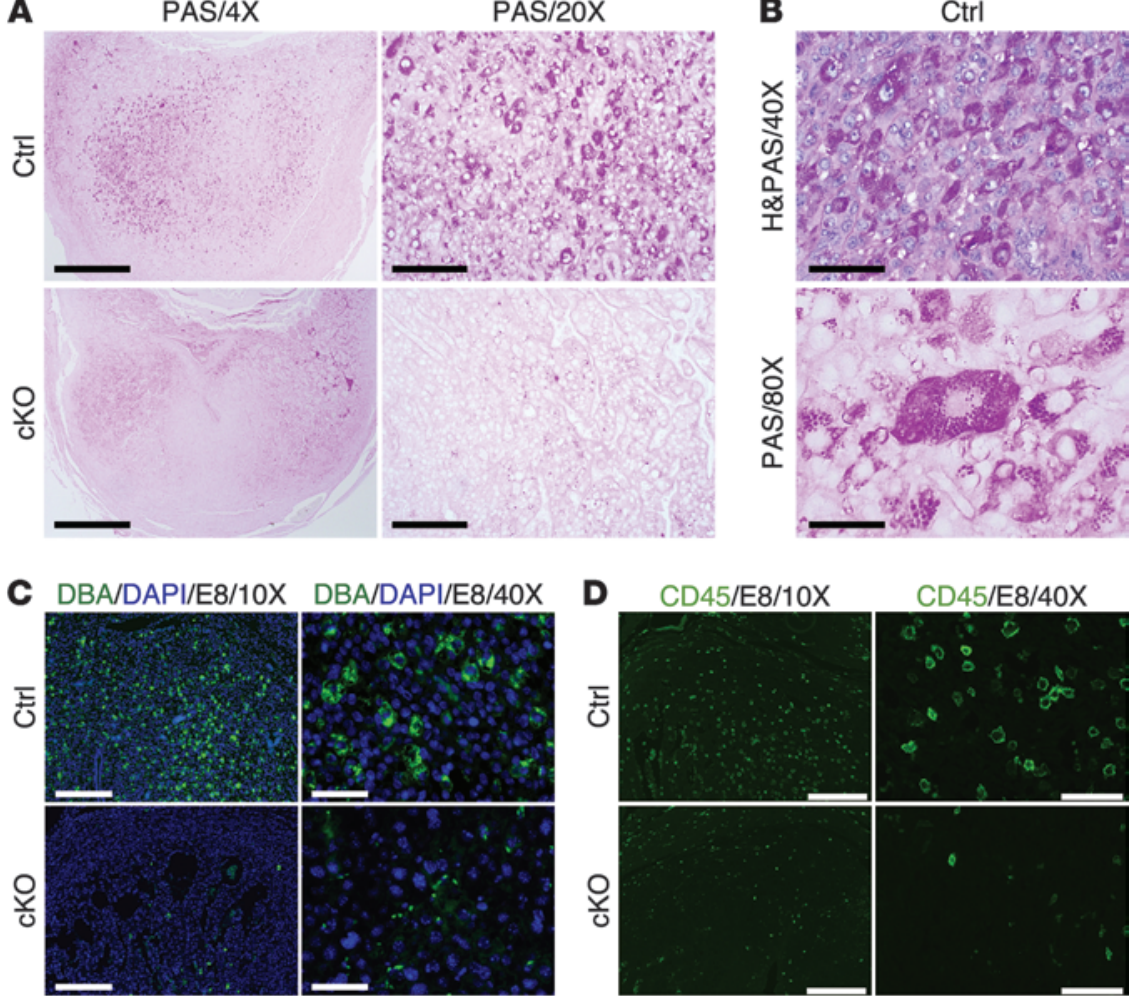

CD45/E8/40X

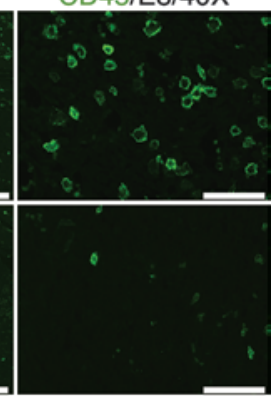

\section{Figure 6}

Deletion of uterine Bmpr2 induces deficiency of UNK cells. ( $A$ and $\mathbf{B}$ ) E9 implantation sites from control and Bmpr2 cKO mice were stained with PAS or hematoxylin-PAS (H\&PAS) and examined for the presence of PAS-positive granulated uNK cells. Scale bar: $1 \mathrm{~mm}(\times 4$ panels); $200 \mu \mathrm{m}$ ( $\times 20$ panels); $100 \mu \mathrm{m}(\times 40$ panel); $50 \mu \mathrm{m}$ ( $\times 80$ panel). (C) Using DBA, uNK cells were abundant in the deciduae of control E8 implantation sites but were rare in E8 deciduae of Bmpr2 cKO implantation sites. Scale bar: $200 \mu \mathrm{m}$ ( $\times 10$ panels); $50 \mu \mathrm{m}(\times 40$ panels). (D) Immunofluorescence analysis using an anti-CD45 antibody detects 6.6 -fold more leukocytes in deciduae of E8 implantation sites of control mice compared with those in Bmpr 2 cKO female mice. Scale bar: $200 \mu \mathrm{m}$ ( $\times 10$ panels); $50 \mu \mathrm{m}$ ( $\times 40$ panels).
E11 (Supplemental Figure 6, E and F). Thus, the placentation defects in Bmpr2 cKO female mice are not attributable to abnormalities in hormone production.

Increased trophoblast giant cells and impaired trophoblast invasion in the implantation sites of Bmpr 2 cKO female mice. Initial trophoblast invasion to establish vascular connections between the fetal ectoplacental cone and maternal decidual tissue occurs around E9 (24). To examine trophoblast formation and function in Bmpr2 cKO female mice at E9, we performed immunostaining for PRL3D1. We found increased PRL3D1-positive cells in uteri of pregnant $\mathrm{Bmpr} 2$ cKO mice compared with those in control uteri, concomitant with disappearance of the normal structure of the trophoblast giant cell layer (Figure 2A). We isolated trophoblast cells (Supplemental Figure 7A) and found that Prl3d1 and $M m p 9$ mRNAs were increased in isolated trophoblast cells from Bmpr2 cKO female mice at E9 (Figure 2B). In contrast, many implantation sites of pregnant Bmpr2 $\mathrm{cKO}$ female mice continued to have remnants of the uterine lumen (Figure 2C), suggesting a defect in the proliferation of decidual cells and/or other cells in the implantation site. While ectoplacental cone cells, positive for the trophoblast marker cytokeratin 8 , were evident at E9 (Figure 2, C and D), there was a suppression of ectoplacental cone markers $\mathrm{Cnr} 1$ and Faab (ref. 25 and Figure $2 \mathrm{E})$. There were also significant increases in TUNEL-positive cells and caspase-3 (Casp3) mRNA levels (1.9-fold elevated) in the trophoblast cells within the ectoplacental cone and invasive trophoblast cells (Figure 2F and Supplemental Figure 7C) in the Bmpr2 cKO female mice. In contrast to the similar PTGS2 expression and expansion of E5 trophoblast giant cells (Supplemental Figure 3E), Bmpr 2 cKO implantation sites at E9 showed a suppression of PTGS2-positive invasive trophoblast cells that are normally present at the initiation of placentation (ref. 25 and Figure 2G). There was also a failure of fetal blood vessels, which are positive for the basement membrane marker laminin, to grow from the allantois in Bmpr 2 cKO female mice at E10 (Figure 2H). Thus, the transition from ectoplacental cone cells to invasive trophoblast cells appears to be defective in uteri of pregnant Bmpr $2 \mathrm{cKO}$ mice, which might be an indirect effect (see below) caused by the deletion of BMPR2 in the uterine decidual cells.

Decidualization, proliferation, and vascularization defects in artificially decidualized uterine horns of Bmpr2 cKO female mice. To investigate the mechanisms of decidual growth restriction in $B m p r 2 \mathrm{cKO}$ female mice and to eliminate possible effects from the fetus and trophoblast cells, we induced an artificial decidual reaction (Figure $3 \mathrm{~A}$ ). One uterine horn was given a decidualization stimulus, while the contralateral horn served as an unstimulated control. We examined uteri on day 6 after artificial decidual stimulation, since mesometrial decidualization (MD) and antimesometrial decidualization (AMD) are maximal after day 7 of pregnancy or pseudopregnancy in female mice (26). Decidual responses were reduced in stimulated Bmpr $2 \mathrm{cKO}$ uterine horns, including a $>50 \%$ reduction in weights (Figure 3, B and C); suppressed differentiation of endometrial stroma cells to epithelioid cells (Figure 3D); lower levels of alkaline phosphatase, a marker for stromal cell differentiation (Figure 3E); and a decreased number of decidualizing stromal cells positive for MKI67, a marker of proliferation (Figure 3F). Immunostaining for PECAM1 (CD31), a marker for endothelial cells and vascularization, demonstrated the presence of large vessels in the decidualized control uterine horn and a lack of major vessel formation in the decidualized Bmpr $2 \mathrm{cKO}$ uterine horns (Figure 3G). However, no obvious differences in apoptosis were detected in the stimulated uterine horns by TUNEL assay (data not shown) and real-time quantitative PCR analysis of Casp3 

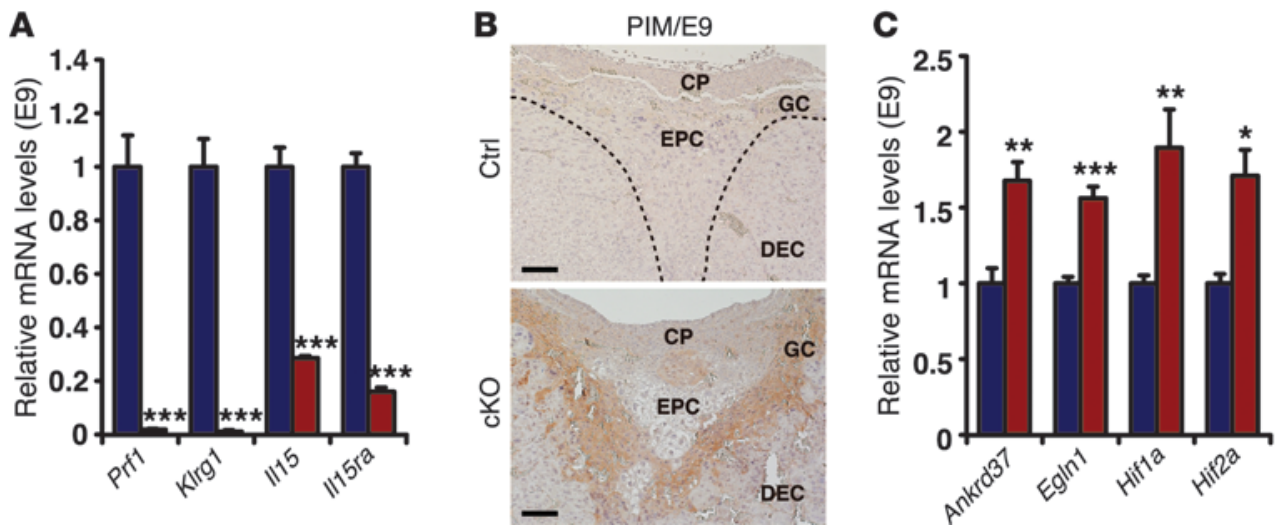

\section{Figure 7}

Altered expression of uNK cell and hypoxia genes in the Bmpr2 cKO uteri. (A) Deletion of uterine Bmpr2 decreases mRNA levels of uNK cellrelated genes in E9 trophoblast cells ( $\left.{ }^{\star * *} P<0.001\right)$. Data are mean \pm SEM. (B) Stimulated hypoxia signaling was detected in E9 trophoblast giant cells within Bmpr2 cKO uteri. The dashed lines represent the border between decidua and ectoplacental cone. Scale bar: 200 um. (C) Deletion of uterine Bmpr2 increases transcription levels of hypoxia-responsive genes in E9 trophoblast cells $\left({ }^{\star} P<0.05 ;{ }^{* \star} P<0.01 ;{ }^{* \star \star} P<0.001\right)$. Data are mean \pm SEM.

(Supplemental Figure 7C). Thus, deletion of uterine Bmpr2 causes differentiation, proliferation, and vascularization defects in the decidual-stimulated uterine stromal cells.

Proliferation and differentiation defects in the decidual zones of pregnant $B m p r 2$ cKO female mice. Since the uterine lumen remains in the decidual zone of E9 implantation sites and artificial decidualization suggests a defect in decidual cell proliferation, we examined decidual cell proliferation and cell cycle regulation in pregnant Bmpr2 cKO female mice. We first analyzed mRNA for Prl3c1 and Prl8a2, markers for decidual cell differentiation $(27,28)$, in isolated decidual tissues from E9 control and Bmpr2 cKO uteri (Supplemental Figure 7B) and found attenuation of these transcripts in uteri of pregnant Bmpr2 cKO mice (Figure 4A). MKI67-positive cells in both MD and AMD compartments at E9 were also decreased by $>50 \%$ (Figure $4, \mathrm{~B}$ and $\mathrm{C}$ ). Flow cytometric analyses revealed a decreased number of $B m p r 2 \mathrm{cKO}$ cells in $\mathrm{S}$ and $\mathrm{G}_{2}$ phases of the cell cycle at E9 (control, 15.9\% $\pm 2.3 \%$; Bmpr2 cKO, $4.7 \% \pm$ $1.1 \%$; Figure 4D) but not at E8 (control, $10.4 \% \pm 2.5 \%$; Bmpr $2 \mathrm{cKO}$, $14.1 \% \pm 3.0 \%$ ). In addition, the mRNA and protein levels of the $\mathrm{G}_{1}$ phase cell cycle regulator, cyclin D3 (CCND3), were statistically lower in decidual tissues within uteri of pregnant $B m p r 2 \mathrm{cKO}$ mice from E9 (Figure 4, E-G, and Supplemental Figure 8, A and B), whereas the mRNA levels of other cell cycle genes were unaffected (Supplemental Figure 8B). CCND3 is implicated in $\mathrm{G}_{2}$ phase arrest in decidualizing stromal cells during formation of polyploidy (4), and AKT regulates CCND3 stability and polyploidy in decidualizing stromal cells (29). However, phosphorylated and total AKT (pAKT and tAKT) levels were unaffected in E9 Bmpr2 cKO uteri (Figure 4G), indicating AKT-independent suppression of CCND3.

Defects in vascular development in uteri of pregnant $B m p r 2$ cKO mice. Since vascular defects were observed in the artificially decidualized tissue from $B m p r 2 \mathrm{cKO}$ female mice, we analyzed the vasculature of pregnant Bmpr $2 \mathrm{cKO}$ female mice. Using an antibody against $\alpha$-smooth muscle actin (actin A2 [ACTA2]), which marks vessels that have smooth muscle cells, we detected expression of the protein in uterine smooth muscle cells and vasculature in the region of the developing spiral arteries within E8 and E9 implantation sites from control female mice (Figure 5A). However, E8 and E9 implantation sites of $\mathrm{cKO}$ female mice showed expression of ACTA2 in the uterine smooth muscle, and vasculature reminiscent of spiral arteries was lacking (Figure 5A and Supplemental Figure 9A). Immunostaining for PECAM1 at E9 also confirmed a paucity of decidual blood vessels in other regions of the decidual zone in the uteri of Bmpr $2 \mathrm{cKO}$ pregnant mice (Figure 5B). Thus, there is a major defect in formation of vasculature and subsequent development of the spiral arteries.

Since VEGFs are potent paracrine stimulators of endothelial cell proliferation and crucial angiogenic factors during decidualization $(30,31)$, we examined Vegf mRNAs in isolated decidual tissue and found reduced levels of Vegfa, Vegfb, and Vegfc mRNA in uteri of pregnant Bmpr $2 \mathrm{cKO}$ mice beginning at E8 (Figure 5C and Supplemental Figure 9B). Angiopoietins (ANGPTs) and their receptors, TIE1 and TEK, are a second vascular tissue-specific receptor tyrosine kinase system to participate in the formation of blood vessels (32). ANGPT1 is a vessel-stabilizing factor, ANGPT2 is a partial antagonist of ANGPT1 and a vessel-branching and permeability factor, and ANGPT4 is a vessel migration factor (33-35). Angpt1 knockout mice die embryonically due to bleeding, concomitant with collapsed atrial endothelial lining (35), and ANGPT2 is readily detectable only in the ovary, placenta, and uterus, the three predominant sites of vascular remodeling in adults (36). We found no obvious defects in Tie1 and Tek mRNA levels (data not shown). Similar to Vegfa mRNA, the three Angpt mRNAs increased from E7 to E9 in the control uteri (Figure 5D and Supplemental Figure 9B). However, Angpt1, Angpt2, and Angpt4 mRNA and protein levels were dramatically reduced in uteri of pregnant $B m p r 2 \mathrm{cKO}$ mice at E8 to E10 (Figure 5D and Supplemental Figure 9, B-D). These findings suggest that BMPR2 either directly or indirectly controls uterine vascular development and subsequent spiral artery remodeling, possibly through suppression of VEGF and ANGPT expression.

Many abnormalities in pregnant Bmpr $2 \mathrm{cKO}$ female mice, such as fetal growth retardation, hemorrhagic implantation sites, and discharge of fetus and placenta, are remarkably similar to human placental abruption. Preeclampsia is a major risk factor for placental abruption (37) and is associated with widespread trophoblast cell apoptosis (38), findings found in pregnant Bmpr2 cKO 
female mice. CORIN, also known as atrial natriuretic peptide-converting enzyme, is detected in pregnant mouse and human uteri (39). Impaired CORIN expression or function in pregnant uteri is associated with preeclampsia, and pregnant Corin knockout female mice with preeclampsia have defects in trophoblast invasion and spiral artery remodeling, similar to our pregnant $\mathrm{Bmpr} 2$ cKO female mice (39). Therefore, we examined CORIN expression between control and Bmpr2 cKO uteri at E9 and discovered that Corin mRNA and protein levels were dramatically decreased in isolated decidual tissues from Bmpr2 cKO uteri (Figure 5E and Supplemental Figure 9C). These results indicate that signaling through BMPR 2 directly or indirectly regulates CORIN expression for the control of trophoblast invasion and therefore could protect against preeclampsia and placental abruption.

Deletion of uterine Bmpr2 prevents differentiation of uterine natural killer cells. To further interrogate the defects in uteri of pregnant Bmpr $2 \mathrm{cKO}$ mice, we investigated the status of uterine natural killer (uNK) cells, potential sources of VEGFs and putative regulators of uterine spiral artery remodeling $(22,40)$. In pregnant mouse implantation sites, uNK cells are the major leukocyte population at E6 to E8 (41) and localize to the mesometrial tissue in the vicinity of the spiral arteries as they differentiate. Immunohistochemical analysis of the E9 implantation sites of the control mice detected a large number of PAS-positive uNK cells with prominent granules (Figure 6, A and B). In contrast, similar PAS-positive uNK cells were absent in the decidual tissue of implantation sites from E9 Bmpr2 cKO female mice (Figure 6A). To confirm this finding, we performed analysis with Dolichos biflores agglutinin (DBA). Precursor NK cells are known to differentiate into granulated uNK cells that are marked by DBA (42). Whereas DBA-positive uNK cells are present in the MD compartment of E8 and E9 control uteri, differentiated uNK cells were essentially absent in pregnant Bmpr2 cKO deciduae (Figure 6C and Supplemental Figure 10A) and artificially stimulated Bmpr2 cKO uterine horns (Supplemental Figure 10B). To further understand this deficiency of uNK cells, we used an antibody for the pan-leukocyte antigen, CD45 (PTPRC), which likely stains predominantly uNK cells and precursors within the E8-E9 decidua basalis. We determined that E8 control implantation sites have 6.6-fold more CD45-positive cells in the MD decidual zone compared with the E8 Bmpr2 cKO implantation sites (control, $832 \pm 247$ cells $/ \mathrm{mm}^{2}$ $[n=3] ; B m p r 2,126 \pm 16$ cells $/ \mathrm{mm}^{2}[n=3]$; Figure $\left.6 \mathrm{D}\right)$. Consistent with these data, mRNAs encoding Prf1, Klrg1, and various granzyme markers for mature and differentiated uNK cells $(43,44)$ were nearly absent in uteri of pregnant Bmpr $2 \mathrm{cKO}$ mice (Figure 7A and Supplemental Figure 10C). mRNA encoding IL-15 (Il15), a regulator of uNK cell differentiation (45-47), and its receptor Il15ra were also severely reduced in uteri of pregnant $B m p r 2 \mathrm{cKO}$ mice (Figure 7A). Our data mechanistically suggest that BMPR2mediated signaling regulates the expression of IL-15 and its receptor or other signaling proteins, which could also alter vasculogenesis, homing of the precursor NK cells into the uterus (27), exit of the NK cells from the vasculature, and/or uNK cell differentiation.

uNK cell depletion not only attenuates uterine spiral artery development but also maternal hypoxia signaling pathways through reduction in the expression of VEGFs (22). Pimonidazole hydrochloride (PIM) forms adducts with proteins under low oxygen tension $(<10 \mathrm{mmHg})$, and these adducts can be detected by immunohistochemical analyses (48). Intensive accumulation of PIM-protein adduct formation was detected in trophoblast giant cells of the ectoplacental cone within uteri of pregnant Bmpr2 cKO mice, indicating low oxygen tension (Figure 7B), and mRNAs encoding the hypoxia-responsive genes $A n k r d 37$ and $\operatorname{Eg} \ln 1(49,50)$ and hypoxia signaling pathway control genes Hifla and Hif2a (50) were increased in isolated trophoblast cells from uteri of pregnant Bmpr 2 cKO mice (Figure 7C). Thus, deletion of uterine Bmpr2 activates the hypoxia signaling pathways in trophoblast cells, likely through defective vasculogenesis and depletion of uNK cells.

\section{Discussion}

BMPs are multifunctional proteins that regulate cell proliferation, differentiation, and apoptosis (11). Over the last decade, production and analysis of mice with targeted deletions of BMP signaling components have uncovered critical roles of BMPs in female reproduction, including follicular development, germ cell-somatic cell interactions, uterine physiology, and cancer $(11,51)$. In further support of the importance of BMP signaling pathways in female reproduction, this study has discovered that uterine BMPR2-mediated signaling controls the formation and function of uterine decidua, which is critical either directly or indirectly for regulating the functions of several downstream pathways essential for postimplantation embryo development and maintenance of pregnancy.

BMP2 has been shown to be a crucial regulator for uterine function. Bmp2 cKO female mice are infertile due to absence of a decidualization response (17). To further dissect the functional requirements of the downstream signaling receptor of BMP2 in the uterus, we generated a cKO of Bmpr2, which encodes a type 2 receptor for BMPs. We found that Bmpr $2 \mathrm{cKO}$ female mice were infertile and did not deliver any live pups during a 6-month fertility test period despite normal implantation. Since BMPR2 is not required for implantation, it cannot be the sole receptor for BMP2, thereby implicating the other type 2 receptors (i.e., ACVR2A and ACVR2B) either alone or redundantly with BMPR2 in this process. The physiological ligands of BMPR2 during pregnancy await further investigation.

Decidualization, a feature of the pregnant uterus, is the process of endometrial cell transformation into secretory cells rich in glycogen and lipid. In response to implantation, uterine stromal cells initiate extensive proliferation and differentiate into decidual cells, which form an avascular primary decidual zone, followed by a well-vascularized secondary decidual zone (4). Using both artificial decidualization and natural pregnancy, we discovered that Bmpr2 cKO female mice exhibited decidual growth restriction, a defect that is potentially linked to dysregulation of CCND3, a key mediator of $\mathrm{G}_{2}$ phase arrest in decidualizing stromal cells (4). Other investigators have shown that AKT regulates CCND3 stability and polyploidy in decidual cells, thereby controlling decidualization (29). However, mRNA levels of Cdk4, Cdk6, and Cdkn1a and protein expression levels of tAKT and pAKT were comparable between isolated decidual cells from uteri of pregnant controls and uteri from Bmpr 2 cKO mice (Figure 4G and Supplemental Figure 8B). Therefore, the involvement of CDK4, CDK6, CDKN1A, and AKT in the regulation of decidual cell proliferation/development in our Bmpr 2 cKO female mouse model is not favored. Nevertheless, our results indicate that BMPR2 is essential for postimplantation decidualization and proliferation of uterine stromal cells, likely through regulation of CCND3.

VEGFs are key angiogenic factors involved in decidualization $(30,31)$, and the reduced uterine expression of Vegf transcripts in our Bmpr $2 \mathrm{cKO}$ model stimulated efforts to identify the proper- 
ties of uNK cells, potential sources of VEGFs $(22,40)$. We demonstrated that uterine BMPR2-mediated signaling is necessary for the presence of uNK cells and development of uterine decidual blood vessels. In our study, vascularization defects and accompanying depletion of uNK cells were detected not only in pregnant uteri but also in decidual stimulated uteri (lacking trophoblast cells) from Bmpr2 cKO female mice. Although previous reports have revealed that uNK cell-depleted female mice made by genetic or pharmacologic methods have narrow lumens of uterine arteries in decidual tissues and reduced VEGF expression $(22,26,27$, $47,52-54)$, these other models do not show severely hemorrhagic implantation sites. These findings suggested that Bmpr2 cKO female mice have other vascularization anomalies.

Furthermore, uNK cells are also recognized as regulators for the hypoxia-trophoblast cell differentiation/invasion pathway (22). Maternal hypoxia during the labile period of placental morphogenesis activates trophoblast giant cells and invasive trophoblast cells, resulting in uterine vascular modification and placental formation (23). Depletion of uNK cells induces trophoblast differentiation and invasion through stimulation of the hypoxia signaling pathways (22). In Bmpr2 cKO female mice, there are defects in the growth of the ectoplacental cone and trophoblast differentiation and invasion and increased apoptosis in the trophoblast cells; since BMPR2 is deleted from the uterine decidua, these extraembryonic defects are likely secondary to the decidual defects that are unfavorable for trophoblast cell invasion (e.g., loss of migration signals for trophoblast cells) and/or the vascular anomalies. Moreover, expression of CORIN, a protein associated with preeclampsia and trophoblast invasion and spiral artery remodeling (39), is dramatically impaired in Bmpr2 cKO female mice. Thus, absence of uterine BMPR2 indirectly affects both uNK cell presence and trophoblast cell invasion, the latter of which is potentially associated with dysregulation of CORIN expression.

ANGPTs are essential for vascular remodeling and sprouting new blood vessels due to their roles in stabilization, branching, permeabilization, and migration of the blood vessels (33-35). ANGPTs are known to be expressed from hematopoietic stem cells, endothelial cells, or periendothelial cells, such as pericytes and smooth muscle cells $(36,55,56)$. In addition, ANGPT1 appears to play an angiogenic role at later time points than VEGFs and distinct from that of VEGFs (55), and ANGPT2 regulates vascular remodeling with ANGPT1 (33). Since BMPR2 is expressed primarily in decidual cells, our results indicate that signaling through BMPR2 secondarily regulates ANGPT expression in endothelial cells.

In summary, we discovered that uterine BMPR2-mediated signaling primarily controls the formation and function of uterine decidua and secondarily controls the development of postimplantation embryos through regulating key pathways (e.g., uNK cell migration and/or differentiation and VEGF, ANGPT, and CORIN signaling) that are essential for uterine vascular development. The distinct phenotypes between female mice with uterine deletion of Bmp2 and Bmpr2 indicate that BMP2 signals through redundant BMP type 2 receptors (i.e., BMPR2, ACVR2A, and/ or ACVR2B) in the implantation period but that BMPs signal nonredundantly through BMPR2 for postimplantation events. Our Bmpr 2 cKO model has defects in uterine decidualization, placentation, and maintenance of pregnancy and mimics intrauterine growth retardation and placental abruption. The Bmpr2 cKO mice may also be exploited for their potential relevance as a model for preeclampsia. Thus, this study has potential implications in developing new BMP-mediated therapies to treat these maternal diseases by targeting the specific genes and pathways involved in these clinical conditions.

\section{Methods}

Generation of Bmpr2 cKO female mice. Mice carrying the Bmpr2-null allele (Bmpr2 $2^{+-}$mice) have been described previously (15). Mice carrying the $B m p r 2$ conditional allele (Bmpr $2^{\ell / / f l}$ mice) were created by flanking $B m p r 2$ exons 4 and 5 , which encode the transmembrane domain and a portion of the kinase domain, with 2 loxP sites (57). The $B m p r 2^{+--}$mice were bred to mice carrying Pgr-cre knockin (Pgrcre/+ mice) (19) alleles to generate $B m p r 2^{+/-}$Pgrre/+ mice. These mice were then bred to $B m p r 2^{f / / f l}$ mice to generate Bmpr $2^{f /-}$ Pgr ${ }^{\text {rre } /+}$ female mice, which were designated Bmpr $2 \mathrm{cKO}$. $B m p r 2^{f l-}$ female mice were controls. Mice were genotyped by PCR analyses of genomic tail DNA using specific primers, as shown in Supplemental Table 2. Analyses of DNA recombination in the uterus were performed using isolated uterine DNA. All mouse lines were maintained on a hybrid C57BL/6J and 129S5/SvEvBrd genetic background.

Fertility analysis. To examine fertility of female mice, WT, Pgrcre/+, control, and Bmpr 2 cKO female mice were mated independently with WT fertile male mice for a 6 -month period ( $n=10$ per genotype). Cages were monitored daily, and the numbers of litters and pups were recorded.

Histological analysis. Ovaries and uteri were dissected and fixed in $10 \%$ neutral buffered formalin for histology. Tissue processing and embedding were performed in the Baylor College of Medicine Department of Pathology Core Laboratory. Paraffin sections $(5 \mu \mathrm{m})$ were stained with PAS, hematoxylin-PAS, or H\&E.

Tissue collection and RNA extraction. Tissues were collected and stored immediately at $4^{\circ} \mathrm{C}$ using RNAlater (Life Technologies) until RNA extraction. Methods for trophoblast and decidual tissue collection were as reported previously $(58,59)$.

Real-time quantitative PCR. Real-time quantitative PCR was performed as described previously (60). Either the SYBR Green detection system or the TaqMan Assays-On-Demand PCR primer and probe sets (Life Technologies) were used for individual gene analysis. Primer information and TaqMan assay ID are listed in Supplemental Table 2. Each sample was analyzed in duplicate, and relative mRNA levels were calculated by the $2^{-\Delta \Delta C}$ method as described previously (61), normalized to the endogenous reference $(G a p d b)$, and plotted as mean \pm SEM.

Immunohistochemistry. Immunohistochemistry was performed as described previously (60). Antibody information is listed in Supplemental Table 3. Tissue sections were briefly counterstained with or without Mayer's hematoxylin. Oxygen tensions at placentation sites were estimated by pimonidazole-protein adduct formation (48) using the Hypoxyprobe Plus Kit (HPI Inc.). To compare intensity of immunostaining between control and Bmpr $2 \mathrm{cKO}$ female mice, tissue sections from both genotypes were developed for the same amount of time. A section without primary antibody was used as negative control.

Immunofluorescence. Paraffin sections were deparaffinized, hydrated, and boiled for antigen retrieval by the same methods as those used for immunostaining. After blocking with 5\% normal serum/PBS for 1 hour at room temperature, tissue sections were incubated overnight at $4^{\circ} \mathrm{C}$ with the primary antibodies. Antibody information is listed in Supplemental Table 3. After washing with PBS, tissue sections were incubated with Alexa Fluor 546- and 488-conjugated secondary antibodies (Life Technologies) for 1 hour at room temperature and mounted in VECTASHIELD HardSet Mounting Medium with DAPI (Vector Laboratories) to visualize chromatin.

TUNEL labeling assay of apoptotic cells. TUNEL assay was performed to analyze DNA fragmentation associated with apoptosis using the ApopTag 
Plus Fluorescein In Situ Apoptosis Detection Kit (Millipore), and slides were mounted in VECTASHIELD HardSet Mounting Medium with DAPI. Immunostaining for ACTB was conducted for identification of structural changes and positional relationship for each tissue compartment.

Western blot analysis. Protein extraction and Western blot analysis were performed as described previously (60). Antibody information is listed in Supplemental Table 3.

Follicle development and cumulus expansion assay. To study follicle development, immature (4-week-old) control and Bmpr2 cKO female mice ( $n=3$ for each genotype) were given intraperitoneal injections of 5 international units pregnant mare serum gonadotropin (Sigma-Aldrich), and ovaries were collected after 46 hours. In vivo and in vitro cumulus expansion analyses were performed as described previously (60). For these analyses, immature (4-week-old) control and Bmpr2 cKO female mice ( $n=3$ for each genotype) were used.

Analysis of superovulation. Superovulation analysis was performed as described previously (60) using 3-week-old control and Bmpr2 cKO female mice.

Analysis offertilization, preimplantation embryo development, and implantation. Control and Bmpr2 cKO female mice were mated with WT fertile male mice to induce pregnancy. The day of vaginal plug detection was considered day 1 of pregnancy (E1). To examine fertilization, female mice were sacrificed at E2, dissected oviducts were flushed with M2 medium (Sigma-Aldrich), and 2 -cell embryos were recovered and assessed. To examine preimplantation embryo development, female mice were sacrificed at E4. After removal and flushing of uteri with M2 medium, blastocysts were recovered and counted. To examine the attachment and implantation reaction, female mice were sacrificed at E5. Implantation sites were visualized using Chicago blue dye.

Hormone assays. Blood was collected from 4-month-old adult female mice by cardiac puncture as described previously (60). Serum follicle-stimulating hormone (FSH), luteinizing hormone ( $\mathrm{LH}), \mathrm{E}_{2}$, and $\mathrm{P}_{4}$ were measured by the University of Virginia Center for Research in Reproduction Ligand Assay and Analysis Core (http://www.medicine.virginia.edu/research/institutes-and-programs/crr/ligand-page).

Artificial induction of decidualization and alkaline phosphatase staining. Artificial decidualization of the uterus and alkaline phosphatase staining were performed as described previously (60).
Cell cycle analysis by using flow cytometry. After centrifugation, isolated decidual cells were mixed with $1 \times \mathrm{RBC}$ lysis buffer (eBioscience) to remove erythrocytes, fixed with $70 \%$ ethanol overnight at $4^{\circ} \mathrm{C}$, and stained with propidium iodide for 30 minutes at $37^{\circ} \mathrm{C}$ for the analyses.

Statistics. All experiments were repeated at least 3 times independently. Differences among groups were assessed by using 2-tailed paired Student's $t$ test or 1-way ANOVA, and the mean between individual groups was further compared using Tukey's honestly significant difference test or Dunnett's test. $P$ values of less than 0.05 were considered statistically significant.

Study approval. Animal handling and surgeries were performed according to the NIH Guide for the Care and Use of Laboratory Animals and were approved by the Institutional Animal Care and Use Committee of Baylor College of Medicine.

\section{Acknowledgments}

We thank the Matzuk laboratory members for helpful advice; Jun Kawagoe for technical advice; and Shirley Baker for aid with manuscript formatting. We are grateful to En Li and Hideyuki Beppu for providing the $B m p r 2 f l / f l$ mice. This research is supported by NIH grants HD032067 (to M.M. Matzuk), R01HD042311 (to F.J. DeMayo), and U54HD007495 (to M.M. Matzuk and F.J. DeMayo) from the Eunice Kennedy Shriver National Institute of Child Health and Human Development and CA077530 (to J.P. Lydon) from the National Cancer Institute.

Received for publication July 9, 2012, and accepted in revised form March 14, 2013.

Address correspondence to: Martin M. Matzuk, Department of Pathology and Immunology, Baylor College of Medicine, One Baylor Plaza, Houston, Texas 77030, USA. Phone: 713.798.6451; Fax: 713.798.5838; E-mail: mmatzuk@bcm.edu.

Takashi Nagashima's present address is: Department of Obstetrics and Gynecology, Keio University School of Medicine, Tokyo, Japan.
1. Welsh AO. Uterine cell death during implantation and early placentation. Microsc Res Tech. 1993;25(3):223-245.

2. Strickland S, Richards WG. Invasion of the trophoblasts. Cell. 1992;71(3):355-357.

3. Glass RH, Aggeler J, Spindle A, Pedersen RA, Werb $Z$. Degradation of extracellular matrix by mouse trophoblast outgrowths: a model for implantation. J Cell Biol. 1983;96(4):1108-1116.

4. Tan J, Raja S, Davis MK, Tawfik O, Dey SK, Das SK. Evidence for coordinated interaction of cyclin D3 with p 21 and cdk6 in directing the development of uterine stromal cell decidualization and polyploidy during implantation. Mech Dev. 2002; 111(1-2):99-113.

5. Georgiades P, Ferguson-Smith AC, Burton GJ Comparative developmental anatomy of the murine and human definitive placentae. Placenta. 2002;23(1):3-19.

6. Pijnenborg R, Vercruysse L, Hanssens M. The uterine spiral arteries in human pregnancy: facts and controversies. Placenta. 2006;27(9-10):939-958.

7. Ball E, Bulmer JN, Ayis S, Lyall F, Robson SC. Late sporadic miscarriage is associated with abnormalities in spiral artery transformation and trophoblast invasion. J Pathol. 2006;208(4):535-542.

8. Kaufmann P, Black S, Huppertz B. Endovascular trophoblast invasion: implications for the pathogenesis of intrauterine growth retardation and preeclampsia. Biol Reprod. 2003;69(1):1-7.
9. Sampath TK, et al. Recombinant human osteogenic protein-1 (hOP-1) induces new bone formation in vivo with a specific activity comparable with natural bovine osteogenic protein and stimulates osteoblast proliferation and differentiation in vitro. J Biol Chem. 1992;267(28):20352-20362.

10. Wozney JM, et al. Novel regulators of bone formation: molecular clones and activities. Science. 1988;242(4885):1528-1534.

11. Chang H, Brown CW, Matzuk MM. Genetic analysis of the mammalian transforming growth factor-beta superfamily. Endocr Rev. 2002;23(6):787-823.

12. Hogan BL. Bone morphogenetic proteins: multifunctional regulators of vertebrate development. Genes Dev. 1996;10(13):1580-1594.

13. Matzuk MM, Lamb DJ. The biology of infertility: research advances and clinical challenges. Nat Med. 2008;14(11):1197-1213

14. Lane KB, et al. Heterozygous germline mutations in BMPR2, encoding a TGF-beta receptor, cause familial primary pulmonary hypertension. Nat Genet. 2000;26(1):81-84.

15. Beppu H, et al. BMP type II receptor is required for gastrulation and early development of mouse embryos. Dev Biol. 2000;221(1):249-258.

16. Delot EC, Bahamonde ME, Zhao M, Lyons KM. BMP signaling is required for septation of the outflow tract of the mammalian heart. Development. 2003;130(1):209-220.

17. Lee $\mathrm{KY}$, et al. Bmp2 is critical for the murine uterine decidual response. Mol Cell Biol. 2007; 27(15):5468-5478.

18. Robker RL, Akison LK, Russell DL. Control of oocyte release by progesterone receptor-regulated gene expression. Nucl Recept Signal. 2009;7:e012.

19. Soyal SM, et al. Cre-mediated recombination in cell lineages that express the progesterone receptor. Genesis. 2005;41(2):58-66

20. Paria BC, Reese J, Das SK, Dey SK. Deciphering the cross-talk of implantation: advances and challenges. Science. 2002;296(5576):2185-2188.

21. Paria BC, Huet-Hudson YM, Dey SK. Blastocyst's state of activity determines the "window" of implantation in the receptive mouse uterus. Proc Natl Acad Sci U S A. 1993;90(21):10159-10162.

22. Chakraborty D, Rumi MA, Konno T, Soares MJ. Natural killer cells direct hemochorial placentation by regulating hypoxia-inducible factor dependent trophoblast lineage decisions. Proc Natl Acad Sci US A. 2011;108(39):16295-16300.

23. Rosario GX, Konno T, Soares MJ. Maternal hypoxia activates endovascular trophoblast cell invasion. Dev Biol. 2008;314(2):362-375.

24. Charalambous F, Elia A, Georgiades P. Decidual spiral artery remodeling during early post-implantation period in mice: investigation of associations with decidual uNK cells and invasive trophoblast. Biochem Biophys Res Commun. 2012;417(2):847-852.

25. Sun X, Xie H, Yang J, Wang H, Bradshaw HB, Dey SK. Endocannabinoid signaling directs 
differentiation of trophoblast cell lineages and placentation. Proc Natl Acad Sci U S A. 2010; 107(39):16887-16892.

26. Rahman MA, Li M, Li P, Wang H, Dey SK, Das SK. Hoxa-10 deficiency alters region-specific gene expression and perturbs differentiation of natural killer cells during decidualization. Dev Biol. 2006;290(1):105-117.

27. Ain R, Trinh ML, Soares MJ. Interleukin-11 signaling is required for the differentiation of natural killer cells at the maternal-fetal interface. Dev Dyn. 2004;231(4):700-708

28. Konno T, et al. Subfertility linked to combined luteal insufficiency and uterine progesterone resistance. Endocrinology. 2010;151(9):4537-4550.

29. Mori M, et al. Death effector domain-containing protein (DEDD) is required for uterine decidualization during early pregnancy in mice. J Clin Invest. 2011;121(1):318-327.

30. Ferrara N, et al. Heterozygous embryonic lethality induced by targeted inactivation of the VEGF gene. Nature. 1996;380(6573):439-442.

31. Halder JB, et al. Differential expression of VEGF isoforms and VEGF(164)-specific receptor neuropilin-1 in the mouse uterus suggests a role for VEGF(164) in vascular permeability and angiogenesis during implantation. Genesis. 2000;26(3):213-224.

32. Augustin HG, Koh GY, Thurston G, Alitalo K. Control of vascular morphogenesis and homeostasis through the angiopoietin-Tie system. Nat Rev Mol Cell Biol. 2009;10(3):165-177.

33. Gale NW, et al. Angiopoietin-2 is required for postnatal angiogenesis and lymphatic patterning, and only the latter role is rescued by Angiopoietin-1. Dev Cell. 2002;3(3):411-423.

34. Lee HJ, et al. Biological characterization of angiopoietin-3 and angiopoietin-4. FASEB J. 2004;18(11):1200-1208.

35. Suri C, et al. Requisite role of angiopoietin-1, a ligand for the TIE2 receptor, during embryonic angiogenesis. Cell. 1996;87(7):1171-1180.

36. Maisonpierre PC, et al. Angiopoietin-2, a natural antagonist for Tie 2 that disrupts in vivo angiogenesis. Science. 1997;277(5322):55-60.

37. Odendaal HJ, Hall DR, Grove D. Risk factors for and perinatal mortality of abruptio placentae in patients hospitalised for early onset severe pre-ec- lampsia - a case controlled study. J Obstet Gynaecol. 2000;20(4):358-364.

38. DiFederico E, Genbacev O, Fisher SJ. Preeclampsia is associated with widespread apoptosis of placental cytotrophoblasts within the uterine wall. $A m J$ Pathol. 1999;155(1):293-301.

39. Cui Y, et al. Role of corin in trophoblast invasion and uterine spiral artery remodelling in pregnancy. Nature. 2012;484(7393):246-250.

40. Hanna J, et al. Decidual NK cells regulate key developmental processes at the human fetal-maternal interface. Nat Med. 2006;12(9):1065-1074.

41. Croy BA, Chen Z, Hofmann AP, Lord EM, Sedlacek AL, Gerber SA. Imaging of vascular development in early mouse decidua and its association with leukocytes and trophoblasts. Biol Reprod. 2012;87(5):125.

42. Paffaro VA, Jr., Bizinotto MC, Joazeiro PP, Yamada AT. Subset classification of mouse uterine natural killer cells by DBA lectin reactivity. Placenta. 2003;24(5):479-488.

43. Allen MP, Nilsen-Hamilton M. Granzymes D, E, $\mathrm{F}$, and $\mathrm{G}$ are regulated through pregnancy and by IL-2 and IL-15 in granulated metrial gland cells. J Immunol. 1998;161(6):2772-2779.

44. Yadi H, Burke S, Madeja Z, Hemberger M, Moffett A, Colucci F. Unique receptor repertoire in mouse uterine NK cells. J Immunol. 2008;181(9):6140-6147.

45. Ye W, Zheng LM, Young JD, Liu CC. The involvement of interleukin (IL)-15 in regulating the differentiation of granulated metrial gland cells in mouse pregnant uterus. J Exp Med. 1996;184(6):2405-2410.

46. Ashkar AA, et al. Assessment of requirements for IL-15 and IFN regulatory factors in uterine NK cell differentiation and function during pregnancy. Immunol. 2003;171(6):2937-2944.

47. Barber EM, Pollard JW. The uterine NK cell population requires IL-15 but these cells are not required for pregnancy nor the resolution of a Listeria monocytogenes infection. I Immunol. 2003;171(1):37-46

48. Samoszuk MK, Walter J, Mechetner E. Improved immunohistochemical method for detecting hypoxia gradients in mouse tissues and tumors. J Histochem Cytochem. 2004;52(6):837-839.

49. Benita Y, Kikuchi H, Smith AD, Zhang MQ, Chung DC, Xavier RJ. An integrative genomics approach identifies Hypoxia Inducible Factor-1 (HIF-1)-target genes that form the core response to hypoxia. Nucleic Acids Res. 2009;37(14):4587-4602.

50. Semenza GL. Oxygen homeostasis. Wiley Interdiscip Rev Syst Biol Med. 2010;2(3):336-361.

51. Shimasaki S, Moore RK, Otsuka F, Erickson GF. The bone morphogenetic protein system in mammalian reproduction. Endocr Rev. 2004;25(1):72-101.

52. Guimond MJ, Luross JA, Wang B, Terhorst C, Danial S, Croy BA. Absence of natural killer cells during murine pregnancy is associated with reproductive compromise in TgE26 mice. Biol Reprod. 1997;56(1):169-179

53. Guimond MJ, Wang B, Croy BA. Engraftment of bone marrow from severe combined immunodeficient (SCID) mice reverses the reproductive deficits in natural killer cell-deficient tg epsilon 26 mice. J Exp Med. 1998;187(2):217-223.

54. Ashkar AA, Di Santo JP, Croy BA. Interferon gamma contributes to initiation of uterine vascular modification, decidual integrity, and uterine natural killer cell maturation during normal murine pregnancy. J Exp Med. 2000;192(2):259-270.

55. Davis $\mathrm{S}$, et al. Isolation of angiopoietin-1, a ligand for the TIE2 receptor, by secretion-trap expression cloning. Cell. 1996;87(7):1161-1169.

56. Takakura $\mathrm{N}$, et al. A role for hematopoietic stem cells in promoting angiogenesis. Cell. 2000;102(2):199-209.

57. Beppu H, Lei H, Bloch KD, Li E. Generation of a floxed allele of the mouse BMP type II receptor gene. Genesis. 2005;41(3):133-137.

58. Kashiwagi A, et al. The postimplantation embryo differentially regulates endometrial gene expression and decidualization. Endocrinology. 2007;148(9):4173-4184

59. Hirota Y, Daikoku T, Tranguch S, Xie H, Bradshaw HB, Dey SK. Uterine-specific p53 deficiency confers premature uterine senescence and promotes preterm birth in mice. J Clin Invest. 2010;120(3):803-815.

60. Nagashima T, et al. Connective tissue growth factor is required for normal follicle development and ovulation. Mol Endocrinol. 2011;25(10):1740-1759.

61. Livak KJ, Schmittgen TD. Analysis of relative gene expression data using real-time quantitative PCR and the 2(-Delta Delta C(T)) Method. Methods. 2001;25(4):402-408. 